\title{
Los escenarios educativos en la actualidad: historicidad, reflexiones y propuestas para la mejora educativa en el Ecuador
}

\section{The current educational scenarios: historicity, reflections and proposals for educational improvement in Ecuador}

\author{
Ericka Figueroa Martínez \\ Universidad de Guayaquil, Ecuador \\ Zila Esteves Fajardo \\ Universidad de Guayaquil, Ecuador \\ Olga Bravo Santos \\ Universidad de Guayaquil, Ecuador \\ Patricia Estrella Asencio \\ Universidad de Guayaquil, Ecuador
}

Autor para correspondencia: ericka.figueroamartinezma@ug.edu.ec, zila.estevesf@ug.edu.ec Patricia.estrellaa@ug.edu.ec,olga,bravos@ug.eud.ec

Fecha de recepción: 28 de Agosto 2017 - Fecha de aceptación: 15 de Octubre de 2017

Resumen: Los escenarios en el proceso educativo, son fundamentales en el desarrollo de la evolución ecuatoriana, la proyección en el tiempo de este causa academia a través de un diseño bibliográfico, las reflexiones que se proponen, es de carácter político puesto que se desarrollan según el espíritu de los gobiernos con influencia externa. Los escenarios han sido creados con los propósitos de los miembros de la comunidad, imaginarios, minorías. El objetivo del presente trabajo consiste en reflexionar acerca de realidades y retos que han impuesto los escenarios educativos, así como generar propuestas para el perfeccionamiento de los procesos educativos en el ámbito ecuatoriano, desde un enfoque de respeto a la diversidad humana y la inclusión educativa.

Palabras claves: escenarios educativos; historicidad y reflexiones; mejora educativa

\begin{abstract}
The scenarios in the educational process are fundamental in the development of the Ecuadorian evolution, the projection in time of this academic cause through a bibliographic design, the reflections that are proposed, is of a political nature since they are developed according to the spirit of governments with external influence. The scenarios have been created for the purposes of the members of the community, imaginary, minorities. The objective of this work is to reflect on realities and challenges imposed by educational scenarios, as well as to generate proposals for the improvement of educational processes in the Ecuadorian sphere, from an approach of respect for human diversity and educational inclusion.
\end{abstract}

Key words: educational settings; historicity and reflections; educational improvement 


\section{Introducción}

Un escenario educativo se construye con la participación activa de actores que cumplen distintos roles y asumen diferentes niveles de compromiso en el proceso educativo (docentes, alumnos, familia y comunidad con sus propios actores). La intervención docente procura pues, participar, mediando entre programas formativos y estudiantes, constituyéndose en un proceso de diseño de situaciones educativas basadas en un sistema de métodos de enseñanza y aprendizaje y educativos, que favorezcan el cumplimiento de los objetivos planteados.

En estos escenarios se produce la formación de los estudiantes expresada en conocimientos, habilidades, hábitos, valores y actitudes, individual y de grupo. Es un proceso complejo, contradictorio, de configuración personal, de relación de lo social y lo individual, del medio más cercano y las experiencias e historia personal, es decir, la educación de niños y niñas, adolescentes, jóvenes y adultos, tiene lugar en la unidad de la actividad y la comunicación, de lo afectivo y lo cognitivo, en un contexto sociocultural dado, donde cada vez más, aparecen nuevos escenarios educativos, que invitan al logro de una mejor preparación y compromiso de los actores formativos para intervenir favorablemente en los procesos de aprendizaje y formación integral de los estudiantes.

Ante los retos que imponen los nuevos escenarios educativos, la dinámica del proceso educativo debe caracterizarse por una educación centrada en los sujetos y el desarrollo del contexto, el protagonismo del estudiante en la construcción de los aprendizajes, la atención, el respeto a la diversidad humana y la inclusión educativa como principio, que posibilita una institución educativa auténticamente abierta a toda la comunidad, respetuosa con las diferencias, de forma que el acceso, la producción, la transmisión, la distribución y la utilización del conocimiento se dé a partir de una perspectiva del respeto al otro, con una actitud de tolerancia, aceptación, cooperación, diálogo y convivencia, garantizando la atención a personas diferentes para lograr el máximo desarrollo de sus capacidades, rechazando cualquier tipo de exclusión educativa, social, económica, cultural, política, étnica, religiosa, de género y favoreciendo la participación y el aprendizaje equitativo.

El objetivo del presente trabajo consiste en reflexionar acerca de realidades y retos que han impuesto los escenarios educativos, así como generar propuestas para el perfeccionamiento de los procesos educativos en el ámbito ecuatoriano, desde un enfoque de respeto a la diversidad humana y la inclusión educativa.

\section{Desarrollo}

Un ambiente de aprendizaje se define como un "lugar" o "espacio" donde el proceso de adquisición del conocimiento ocurre. Es un ambiente de aprendizaje el participante actúa, usa sus capacidades, crea o utiliza herramientas y artefactos para obtener e interpretar información con el fin de construir su aprendizaje. Es un espacio organizado y estructurado de tal manera que facilita el acceso al conocimiento, genera actividades y relaciones que motivan a aprender, en esta línea de ideas es ilustrativa la siguiente cita: "La actividad permite no solamente abordar la realidad del mundo material y transformarlo, sino intercambiar informaciones, es decir, comunicarse con el otro, con los otros”. (González y Flores, 1997: 15). 
Esta definición reduce el concepto ambiente de aprendizaje al espacio o lugar en que tiene lugar la formación y no reconoce el entramado de relaciones que allí se desarrollan, las condiciones económicas, físicas, culturales, sociales que existen, los materiales de apoyo, recursos e implementos que generan análisis, reflexión y apropiación, experiencias y vivencias.

Es por ello que se coincide con Chaparro, (1995), el cual plantea que:

"No se limita a las condiciones materiales necesarias para la implementación del currículo, cualquiera que sea su concepción, o a las relaciones interpersonales básicas entre maestros y alumnos. Por el contrario, se instaura en las dinámicas que constituyen los procesos educativos y que involucran acciones, experiencias y vivencias por cada uno de los participantes; actitudes, condiciones materiales y socioafectivas, múltiples relaciones con el entorno y la infraestructura necesaria para la concreción de los propósitos culturales que se hacen explícitos en toda propuesta educativa." (Chaparro, 1995: 2).

De esta forma, Ortiz, (2011), explicita tres componentes de los ambientes de aprendizaje, estos son:

El escenario, "es el espacio como eje fundamental que reúne aspectos como: ventilación, iluminación, distribución y organización del mobiliario y del material de apoyo, también incluye lo relacionado con el propósito de la enseñanza. Determina la actuación de los sujetos, por ello, facilita la enseñanza, genera el aprendizaje y el desarrollo de los sujetos en su condición individual y colectiva." Los actores, "son los estudiantes y el educador, estos sujetos estimulan su desempeño al entrar en contacto con los otros y consigo mismo. Los contenidos, son la esencia y el pretexto para estructurar determinados ambientes de aprendizaje. Los escenarios educativos cada vez son más cambiantes dado los objetivos que se persiguen con el proceso formativo. En la actualidad, se hace énfasis en los escenarios áulicos, virtuales y reales; pero el éxito se obtiene cuando en este proceso los escenarios educativos se combinan.

Tanto Chaparro como Ortiz indican en sus escritos la importancia de la socio parental y entorno educativo como ejes fundamentales para el desarrollo del proceso de enseñanza y aprendizaje pues el desempeño y desarrollo de los proyectos permite la viabilidad al acceso educativo desde la academia, investigación y la gestión social del conocimiento, inclusive los proyectos de inversión educativa tienen aristas pre establecidas en el área de la inclusión, ecología humana, tecnologías educativas, vinculación con la comunidad.

Por la importancia que revisten en la calidad de la formación y en la producción del hombre nuevo se realiza un análisis reflexivo de los diferentes escenarios educativos que han caracterizado al Ecuador, sobre la base de tres supuestos esenciales:

La epistemología del Sur de Bonaventura de Sousa Santos, referida esencialmente a tres ideas básicas: 1) el capitalismo y el colonialismo continúan profundamente entrelazados, aunque las formas de articular hayan variado a lo largo del tiempo; 2) las prácticas del conocimiento permiten intensificar la voluntad de transformación social y 3) no habrá justicia 
social global sin justicia cognitiva global.

El paradigma de la complejidad, sistematizado por Edgar Morín en el que se jerarquizan el fortalecimiento de la persona valores, identidad, cultura, ciudadanía, convivencia armónica; las competencias genéricas (pensamiento complejo, crítico y creativo, comunicación oral, escrita, digital y simbólica, articulación de saberes, trabajo en equipos colaborativos, aprendizaje autónomo y participación en redes); y, los desempeños profesionales basados en la gestión del conocimiento (investigación, comprensión de problemas y tensiones) conjeturas, indagación y exploración del conocimiento, diseño de sistemas conceptuales y variables, especulación y reflexión crítica, modelos de intervención y manejo de protocolos de proyectos de investigación, desarrollo e innovación tecnológica y social).

La Pedagogía de la esperanza de Paulo Freire, en la que reescribe la pedagogía del oprimido, reflexiona sobre ella, sus ideas, el contexto donde éstas se desarrollaron y tomaron forma, defiende que la educación debe tratar de abrir paso a la verdad, habla de la esperanza como una necesidad ontológica, pero que no es suficiente, resalta la importancia de que no se trata de educar al pueblo, sino de educarse con él, se refiere a la complejidad de la personas y al poder la imaginación y del lenguaje, entiende a la educación como un psicoanálisis histórico, sociocultural y político, que debe partir de los educandos, y no del educador, habla sobre la necesidad de un educador preparado para el diálogo, para que sus alumnos defiendan sus propias ideas y respeten las de los demás, entre otros aspectos de interés.

¿Por qué la educación en el Ecuador no se ha desarrollado como los planes o currículos programáticos establecidos preveían? ¿Cuáles han sido los errores del Estado como administrador del proceso educativo del país? ¿Qué se está haciendo y qué se debe mejorar para cumplir con los estándares internacionales educativos?

A finales del siglo XIX y principios del XX no se percibían avances en el sistema educativo ecuatoriano en el que las inversiones para el sector público se destinaban al mejoramiento de la infraestructura, más que a la creación de planes y programas ejecutables, primaban las relaciones sociales con funcionarios públicos para acceder a ejercer como docente y también se apreciaba el problema de la alfabetización y las brechas entre la educación rural y urbana, para los que se realizaron determinadas acciones que permitieron reducir los índices de afectación, así como incumplimientos de los currículos, pero no fue suficiente.

Todo ello, trajo consigo, poco reconocimiento social de las instituciones públicas y la consecuente emigración de los estudiantes hacia instituciones privadas que tenían evidentes fines lucrativos. Para esa época, el sistema educativo se fortalecía en inequidad donde la calidad de enseñanza se demostraba solo en la capital.

En 1963, en tiempos de la Junta Militar se crea un Programa Nacional de Alfabetización y Educación para Adultos, que rápidamente empieza a dar resultados esperanzadores en el país, pero, por cuestiones políticas, los resultados posteriores no fueron alentadores y más bien se dedicaron a cerrar universidades que no respondían a los intereses 
de la junta (direccionada por la CIA); las que levantaron voces de protesta, como es el caso de la Universidad Central del Ecuador, institución que cerraron en tres ocasiones. La relación educación - sociedad, no se favoreció, ni en su carácter funcionalista, en tanto la educación no generaba desarrollo social, al ritmo que realmente se necesitaba.

A finales del siglo XX, la población rural tuvo la mitad de escolaridad formal que la urbana y una tasa elevada de analfabetismo, marcando visibles diferencias entre la ciudad y el campo. En 1998, se creó una nueva norma jurídica ecuatoriana que establecía que la educación estaba garantizada por el Estado en igualdad de condiciones y oportunidades, que era un derecho irrenunciable de las personas, que sería ética, pluralista, democrática, humanista y científica; incluso se garantizaba la educación para personas con discapacidad, otorgando la libertad de enseñanza y cátedra, fortaleciendo prioritariamente las zonas rurales y fronterizas, garantizando la educación particular sin fines de lucro y asignando no menos del treinta por ciento de los ingresos corrientes totales del gobierno central, para la educación y la erradicación del analfabetismo.

Lo anterior no se cumplió a pesar de la aprobación de un conjunto de normas, leyes y regulaciones y haber generado una red de colegios para favorecer los ingresos, ello estuvo dado por la carencia de docentes preparados para desarrollar las motricidades de los niños (as) en la Educación Inicial y por no completar los programas establecidos en la Educación Básica, entre otros aspectos; existía una evidente desorganización en estas instituciones.

Aunque el clamor internacional era satisfacer las necesidades básicas de aprendizaje garantizando la universalidad del acceso a la educación, para el que se definieron métodos y estrategias en función de satisfacer estas necesidades en 10 años, Ecuador hasta el 2002, era uno de los países que ni siquiera había podido entrar en el proceso de educación preescolar, ya que no se habían creado currículos educativos dirigidos a conseguir estos propósitos, afectando la inclusión educativa y su universalización.

En el año 2006, fue aprobado un nuevo plan decenal 2006-2015, a partir del cual se debía lograr calidad educativa con equidad, interculturalidad e inclusión, desde un enfoque de los derechos y deberes para fortalecer la formación ciudadana y la unidad en la diversidad de la sociedad ecuatoriana.

En el 2007 el escenario educativo toma un rumbo diferente, debido a los aportes generados por el aumento del precio del barril de petróleo y a las políticas progresistas del gobierno de Correa que cambiaron al Estado en sí, siendo la educación, una de las más beneficiadas. Ello se corrobora con las siguientes mejoras:

- Se crea un nuevo currículo nacional.

- Se concibe a la educación como un deber del Estado, así como su gratuidad hasta el tercer nivel de educación superior, lo que revolucionó el sistema educativo.

- Designación de todas las personas que conforman el aparato administrativo en educación en todos sus niveles; se han producido miles de nombramientos en base a concursos libres de méritos y oposición.

- La evaluación y capacitación de docentes para generar una equiparación entre lo que 
propone el currículo y el perfil de los docentes para cumplirlo.

- Se creó un sistema jurídico que favoreció la educación intercultural, la igualdad, la equidad y la inclusión, en el que se destacan la LOEI y la LOES; estas permiten regularizar ciertos aspectos legales sueltos de otras épocas.

- La creación de la Secretaria Nacional de Educación Superior Ciencia, Tecnología e Innovación (SENESCYT) que permitió salvar la Educación Superior que se había convertido con grandes excepciones en instituciones de negocios de pases y obtención de títulos, con planes no cumplidos, formando profesionales con altos índices de vacíos que deberían haber sido llenados por las universidades; ello, obligó a los docentes a realizar estudios de cuarto nivel que avalen su designación como catedráticos de Instituciones de educación superior.

También quedaron cuestiones objeto de análisis, como:

- La generación de un currículo rígido para la Educación Inicial, la EGB y el BGU que los docentes deben cumplir con destrezas establecidas que no permiten el descubrimiento y desarrollo de nuevas destrezas de un proceso dinámico, como lo es el educativo.

- La exigencia de las Tecnologías de la Información y las Comunicaciones (TICs) sin adecuar a las instituciones públicas de estas tecnologías, creando una desigualdad entre instituciones y sobre todo entre estudiantes con el mismo currículo.

- La evaluación está centrada en los resultados y no en el proceso, sin tener en cuenta los ritmos de aprendizaje de los estudiantes, se caracterizan por diseñar y aplicar pruebas estandarizadas.

- Se evidencia dicotomía entre teoría y práctica, expresada en la desvinculación de los contenidos de la práctica y de la vida; por lo que los estudiantes no aprenden teorías que los lleve a comprender la realidad y generar conocimientos.

Todo ello atentó contra la calidad educativa.

La aprobación y puesta en práctica en el 2008 de la Constitución de la República del Ecuador (por Decreto legislativo 0, Registro Oficial 449 de 20-oct-2008, Última modificación: 13-jul-2011), reforzó el derecho del Buen Vivir, y con ello, un mejor tratamiento a los Derechos de las personas y grupos de atención prioritaria, así como de las comunidades, pueblos y nacionalidades.

En el Art. 11 de la Constitución, se plantea que todas las personas son iguales y gozarán de los mismos derechos, deberes y oportunidades: Nadie podrá ser discriminado por razones de etnia, lugar de nacimiento, edad, sexo, identidad de género, identidad cultural, estado civil, idioma, religión, ideología, filiación política, pasado judicial, condición socioeconómica, condición migratoria, orientación sexual, estado de salud, portar VIH, discapacidad, diferencia física; ni por cualquier otra distinción, personal o colectiva, temporal o permanente, que tenga por objeto o resultado menoscabar o anular el reconocimiento, goce o ejercicio de los derechos. La ley sancionará toda forma de discriminación.

En el Artículo. 27, se plantea que - La educación se centrará en el ser humano y 
garantizará su desarrollo holístico, en el marco del respeto a los derechos humanos, al medio ambiente sustentable y a la democracia; será participativa, obligatoria, intercultural, democrática, incluyente y diversa, de calidad y calidez; impulsará la equidad de género, la justicia, la solidaridad y la paz; estimulará el sentido crítico, el arte y la cultura física, la iniciativa individual y comunitaria, y el desarrollo de competencias y capacidades para crear y trabajar.

Las niñas, niños y adolescentes tendrán derecho a su desarrollo integral, entendido como proceso de crecimiento, maduración y despliegue de su intelecto y de sus capacidades, potencialidades y aspiraciones, en un entorno familiar, escolar, social y comunitario de afectividad y seguridad. Este entorno permitirá la satisfacción de sus necesidades sociales, afectivo-emocionales y culturales, con el apoyo de políticas intersectoriales nacionales y locales.

Tales propósitos, quedaron plasmados en los objetivos del Plan Nacional del del Buen Vivir 2009- 2013 y 2013-2017, a través de la proyección de lineamientos y políticas que apuntaron hacia la igualdad, la integración y la cohesión social del individuo, sustentado en una mejora continua del sistema educativo; la rendición de cuentas del Ministerio de Educación del año 2015, permite ilustrar avances educativos que son expresión de la atención a los nuevos retos de los escenarios educativos, por ejemplo:

- La tasa neta de asistencia a Educación General Básica (EGB) alcanzó un 96,3\% en 2015, con lo que se logró mantener la universalización de EGB obtenida en 2011.

- 140 instituciones que ofertan Educación Inicial (EI), 1 por cada Distrito, se constituyeron como referentes pedagógicas y de reorganización de ambientes de aprendizaje, lo que ha permitido fortalecer el desempeño de las instituciones que ofertan este nivel educativo.

- La tasa de asistencia a Educación General Básica de personas auto-identificadas como indígenas (entre 5 a 14 años) creció 8 puntos porcentuales de 2006 a 2015; y la tasa de

- asistencia a Bachillerato de personas auto identificadas como indígenas (15 a 17 años) creció en 31 puntos porcentuales en el mismo período.

- La tasa neta ajustada de asistencia a Bachillerato alcanzó un 68,93\% en 2015, es decir, 15,55 puntos porcentuales más que en 2007 y 3,06 puntos porcentuales más que en 2014.

- En el período lectivo 2014-2015 (Inicio) el número de estudiantes matriculados en educación Intercultural Bilingüe fue 1,6 veces más que en el año 2006, es decir, 53.485 estudiantes más que en el periodo anterior.

- En el 2015 el sistema educativo atendió a 725 estudiantes con necesidades educativas especiales asociadas a una discapacidad, lo que sumado a gestiones anteriores ha permitido llegar a un total de 70156 personas, logrando cubrir al 87,3\% de esta población.

- Construcción e implementación de la Unidad de Educación Especial Manuela Espejo, en la ciudad de Guayaquil, destinada exclusivamente a la atención educativa para personas con discapacidad.

- El país cuenta con 58 Unidades Educativas del Milenio (UEM) que se encuentran en 
funcionamiento, de las cuales 7 se inauguraron en 2015. A estas se sumarán 54 UEM cuya construcción inició en dicho año.

- Al 2015 se impartieron 33,46 millones de horas de capacitación a docentes. Lo que les permitirá incrementar sus conocimientos, destrezas y desempeño al momento de impartir clases.

Estos y otros resultados, dan cuenta de la gestión integral del Ministerio de Educación en Ecuador, sin embargo, temas como: la calidad, la cobertura y la gestión continuaron siendo desafíos para el 2016, donde se connotó el término comunidad educativa, como una filosofía y una práctica más integral de atender los retos de los nuevos escenarios educativos. Algunos de los desafíos fueron:

- Fortalecer la ciencia, la tecnología, la investigación, la innovación y el emprendimiento en todos los niveles y tipos de educación.

- Desarrollar el Programa de Corresponsabilidad Social con la Educación, "Educatón, todos por la Educación” para construir, generar y alcanzar espacios de participación activa con diferentes actores, sectores productivos y agentes externos que quieran sumarse a contribuir en la sostenibilidad y transformación de la educación del país.

- Promover el incremento de uso de la plataforma Comunidad Educativa en Línea (CEL) por parte de la comunidad educativa

- Dotar de infraestructura tecnológica, informática y de conectividad a las instituciones educativas de sostenimiento fiscal.

La convergencia con los objetivos, lineamientos y políticas del Plan Nacional del Buen Vivir 2013- 2017, han permitido seguir avanzando en el tema de la calidad educativa, y su proyección hasta el 2025 en el Ecuador.

En la actualidad, hay una tendencia hacia los escenarios virtuales o hacia el empleo de las Tecnologías de la Información y las Comunicaciones (TICs), como método y medio de enseñanza- aprendizaje y como vía para el análisis y valoración de resultados; precisamente con la llegada de las TICs, la formación educativa da un giro, cambiando diversas situaciones, sobre todo para aquellos que tienen en sus manos la gran tarea de educar. En el sistema educativo se encuentran muchas dificultades originadas en el proceso enseñanza aprendizaje, uno de ellos es la falta de infraestructura tecnológica, por ello es evidente que existen deficiencias en los docentes en "cómo enseñar" y, de igual manera, persisten en "qué enseñar".

Según Fernández (2007) “....no cabe duda de que los usos de estas Tic en los ambientes educativos están transformando la ecología del aula y las funciones del docente, induciendo a mutaciones sistemáticas en las teorías y prácticas didácticas”.

En la actualidad, se habla del síndrome de la tecnología educativa, tal como el investigador Dr. Justo Chávez lo menciona en su aporte investigativo: Actualidad de las Tendencias Educativas, en dónde hace énfasis en que el auge actual y la versatilidad de la tecnología, hace que las propuestas de utilización se sitúen en todas las etapas del sistema de educación, desde el preescolar hasta la universidad. (J. Chávez, 2003). 
De lo anterior, se deriva que el desarrollo tecnológico actual está situando a las organizaciones educativas ante un nuevo paradigma en el proceso de enseñanza aprendizaje, dando lugar a nuevas metodologías y nuevos roles para el docente; desde esta perspectiva, se asume un enfoque humanista en las relaciones actividad- comunicación, que se da entre docentes y estudiantes, mediados por el uso de las TICs; comprendido así, se supera la visión pragmática- tecnologicista en el uso de las funcionalidades de las TICs en el proceso de enseñanza-aprendizaje y educativo, en general.

Otro rasgo a considerar en materia de escenarios a educativos es el referido a la convivencia escolar; que se ha convertido en un reto educativo de primer orden y los centros docentes, como escenarios donde los estudiantes amplían y consolidan sus relaciones interpersonales - a modo de microcosmos social—, pueden y deben cumplir un papel primordial en el aprendizaje de las competencias personales y sociales, tal y como se hace alusión en reportes investigativos dados por autores tales como: Boqué, (2004 $)$; Boqué, (2004b); García y López (2010), citados por Fuensanta Hernández Pina y Javier J. Maquilón Sánchez (2010).

\section{¿Qué hacer para mejorar el proceso formativo ante los nuevos retos que imponen los escenarios educativos en el Ecuador?}

El compromiso para y con el cambio educativo, liderado por el perfeccionamiento continuo de la preparación pedagógica y didáctica de los docentes, con apertura a la innovación educativa y didáctica, a partir del desarrollo de un pensamiento creador, independiente, flexible y alternativo. Una de las claves de la innovación educativa reside en la interrelación cuantitativa y cualitativa que se logra entre lo que se aprende dentro y fuera de la institución escolar, entre la transferencia de conocimiento en la educación formal, no formal e informal. El aula sigue siendo el espacio privilegiado de aprendizaje innovador aunque precisa, cada vez más, del concurso colaborativo de otros escenarios, tiempos y medios: el de los encuentros colectivos de la comunidad escolar; el de las actividades extraescolares y de ocio; el de los artefactos digitales y virtuales; el del acompañamiento familiar en la vida cotidiana de la infancia y la juventud; y el de otras oportunidades educativas, espontáneas u organizadas, que brinda el territorio. Así, el aula ocupa la centralidad de una red de espacios interconectados, pero no es el único escenario educativo.

La diversificación de los escenarios educativos, que favorezca las conexiones y coordinaciones entre las agencias y agentes educativos para el desarrollo del aprendizaje experiencial vivencial y de las relaciones sociales entre docentes-docentes, docentes-alumnos, docentes-educadores sociales, de apoyo y especializados y alumnos-comunidad, ampliando las funciones y representantes de los consejos escolares, creando consejos educativos territoriales u otras dinámicas y estructuras democráticas más participativas. Al respecto, Emilio Lledó planteó: "Lo importante del profesor no es ganarse la vida sino ganar la vida de los demás".

El tránsito de una didáctica tradicional, centrada en el docente y descontextualizada de la realidad y de la vida hacia una didáctica integradora de las ciencias, centrada en el alumno, que enseña a aprender a aprender y exige de un pensamiento y una práctica interdisciplinaria en integración con los saberes ancestrales y de igualdad de género e interculturalidad. 
La promoción de un cambio profundo en la metodología educativa, la cual no consiste en utilizar las nuevas herramientas con métodos tradicionales, sino que se debe modificar a la enseñanza en pro de las necesidades individuales del alumno, a través de la interactividad, creando un nuevo marco de relaciones, fomentando el trabajo colaborativo, ofreciendo una metodología creativa más cercana a la diversidad y a las necesidades educativas especiales.

La necesidad de mantener el acceso libre, sin restricciones de tiempo y espacio al proceso educativo con el uso de herramientas que optimizan el aprendizaje y estimulan a los alumnos que con uso de multimedia, internet y redes sociales han configurado un nuevo mundo de información.

La gestión del cambio en los contenidos didácticos si modificamos métodos, procesos y objeto educativo es claro que el contenido de la educación será modificado por uno más exigente y adecuado a la globalización actual, con más participación, protagonismo y activación del pensamiento lógico de los estudiantes, los que deben desarrollar el sentido crítico y reflexivo.

La Infraestructura de los Centros Educativos debe mejorarse en función de la incorporación de las TICs en el proceso educativo, deberá contarse con el equipo necesario además del espacio físico adecuado para conservarlo. En este cambio se incluyen: Infraestructuras, equipos, gestión, formación del profesorado y ampliación del entorno educativo.

El respeto y estimulación de la convivencia intergeneracional que favorezca las conexiones y vínculos entre alumnos de distintas edades, de intercambio y colaboración entre la infancia, la juventud y la tercera edad para compartir distintos saberes, visiones y experiencias. Asimismo, la diversidad generacional contribuye a enriquecer la dinámica de los equipos docentes al poder compartir los nuevos aportes de la formación inicial con la dilatada experiencia de aula, además del saludable contraste de distintas maneras de pensar y estar en la educación.

Las universidades del país desarrollan estrategia de soluciones que permiten el mejoramiento institucional de las IES, la calidad está en relación con el perfil de salida de los estudiantes que arrojan las diferentes organizaciones, el estudio se propuso valorar el aporte que ha hecho el sistema al mejoramiento de la calidad de aspectos que inciden el desempeño de los estudiantes, en la formación, en la investigación, lo cual hace referencias a la oferta laboral del país.

(Sarango, Prácticas Educativas Abiertas: experiencias de innovación en una institución de educacion superior del Ecuador) "Las universidades necesitan involucrarse en un proceso de cambio y de mejora de la calidad. Lo que quiere decir, promover procesos de innovación docente y de aprendizaje apoyados por las Tecnologías de la Información y Comunicación”

(Xiomara Velez, 2016) Los resultados obtenidos en la presente revisión muestran que, tanto en los planes de estudio de Ecuador como en los planes de estudio de España, la formación 
de los futuros educadores en el ámbito de la educación inclusiva es claramente insuficiente. En el caso de Ecuador, las carreras de Educación Inicial, Educación Básica y Psicología Educativa son las que tienen el mayor número de asignaturas relacionadas con el tratamiento de la inclusión en sus programas, pero la media de materias por carrera es apenas de tres a cinco. El resto de carreras todavía tienen menos asignaturas en materia de inclusión, siendo la media de entre una a ninguna asignatura. En España encontramos una distribución similar a la de Ecuador, siendo los programas de Magisterio de Educación Infantil, Educación Primaria, Pedagogía Terapéutica, Audición y Lenguaje, Educación Física y Lenguas extranjeras las que presentan una media de entre tres a cinco asignaturas relacionadas con la inclusión. No obstante, considerando todas las carreras, en España el porcentaje de programas que tienen al menos una asignatura relacionada con la inclusión es superior al de Ecuador, siendo del 59,44\% en España frente al 36,5\% en Ecuador.

Las mejoras en el ámbito de la Educación Inclusiva en el Ecuador son significativas, en estos últimos diez años las políticas con programas educativos van en aumento los mismos que generan espacios oportunos que incurren en el desarrollo de nuevas tecnologías de la información e inserción laboral, este tipo de educación en su malla la tiene incluida todas las carreras de la Facultad de Filosofía, Letras y Ciencias de la Educación y el programa que se desarrolla en la gestión de Bienestar Estudiantil incluye un trato diferencial en lo académico y en la gestión de recursos destinados para ayudas económicas.

En estudios recientes de (Celia Sarango Lapo et al ,2018) indica en su tesis doctoral: En este estudio se analizaron las formas de implementación de las PEA en tres experiencias en la UTPL, con el fin de identificar elementos clave para la mejora de las prácticas educativas abiertas como instancias de innovación. En primer lugar, en el modelo OpenUTPL los datos mostraron que los profesores no subieron todos los REA que se les solicitaron inicialmente. Las razones se deben, entre otras, a que la mayoría de los docentes en el año 2008 sumaron a sus actividades los estudios doctorales, actividad impulsada como prioritaria por la universidad, lo que les implicó mayor demanda de tiempo en investigación.

La implementación de la modalidad doctoral hizo como requisito que el docente tenga que conjugar su mayor esfuerzo al reconocimiento de su esfuerzo por un documento que los acredite como o docentes investigadores, esta modalidad de estudios a distancia permite al docente actual como ya lo indicamos desde el 2008 con mayor énfasis en plataforma conectarlo en tiempo y espacio.

(Ximena Velez Calvo et al, 2016) Indica que: Las ideas y principios de la inclusión educativa no pueden llevarse a la práctica sin la participación de los docentes, quienes tienen que asumir, junto con las familias, la responsabilidad de promoverlos, gestionarlos e implementarlos. Para ello, sea cual sea el nivel educativo en el que desarrollan su labor, los profesionales de la educación deben tener conocimientos básicos en atención a la diversidad.

Los esfuerzos desde todas las aristas para desarrollar programas que comparezcan en la defensa de la educación inclusiva sobre todo en la Educación Superior ya que los departamentos de Bienestar Estudiantil afianzan los programas de Bienestar Estudiantil Semestre 2018 de la Facultad de Filosofía, Letras y Ciencias en donde los estudiantes por carencias económicas 
fueron beneficiados 63 estudiantes, con discapacidad (enfermedad catastrófica) 1 estudiante, excelencia académica 162 estudiantes, se demuestra en una población de más de mil estudiantes que el desarrollo va en relación de la inclusión y beneficio a los necesitados.

Por otra parte, aún no se ha descubierto la fórmula capaz de superar la crisis, ni se ha determinado con precisión el estado del arte en cuanto a los avances y limitaciones de los programas implementados últimamente; tampoco se han dibujado las líneas del nuevo Plan Decenal ni se dispone de recursos económicos y financieros ni de actores capaces de dar un vuelco a la educación. Pero solo a partir del diagnóstico y un amplio diálogo sobre los logros alcanzados y sobre las limitaciones aún presentes es que tocará diseñar la nueva propuesta, unir voluntades y levantar paradigmas para la próxima década: 2013-2020.

Los esfuerzos por la década ganada ha sido desde fondo y no forma como lo hemos visto a lo largo de la historia en donde la desconcentración sólo ha sido producto magistral de un discurso de izquierda, pero en la dura derecha no ha sido sino una ilusión óptica de una falsa ilusión compartida por coidearios del mismo sistema.

(Edgar Isch López, 2011) Indica que: Los mecanismos de evaluación, en todo caso, retornaron a los procedimientos estandarizados que desconocen la diversidad de condiciones y de sectores sociales ecuatorianos. Esto es más grave si se considera que "las inequidades más grandes encuentran entre alumnos de diferentes niveles socio-económicos" y que "los alumnos de etnias minoritarias también continúan en desventaja ...Su utilización, sin embargo, ha significado el uso de otro tipo de instrumentos de segregación, entre los cuales a fines de 2010 estuvo el proceso de matrícula en octavo año de educación general básica sólo para los que tenían altas calificaciones, relegando a quienes no las tenían, sin considerar en absoluto las razones que, por ejemplo llevan a tener calificaciones menores a los niños que trabajan en media jornada.

Las autonomías propias de las ideologías y tendencias indican que la inclusión es necesario para el proceso del desarrollo hegemónico de una nación, pero el mismo se ve empañado con el desarrollo de proyectos que poco se motivan en la verdadera universalización de los proyectos que están generados en el desempeño de la funcionalidad de dichos documentos, como por ejemplo el proyecto "Aprendiendo en movimiento" que es un modelo no inclusivo por la modalidad de la guía para alumnos regulares más no que presentan una discapacidad o necesidad educativa especial.

Las instituciones se imponen con tendencias administrativas como las que se dan en las empresas privadas por ejemplo tener regulado Plan de Prevención de Riesgo.

(Educación, 2013) Indica que: La reapertura de instituciones educativas o de espacios de aprendizaje lo más rápidamente posible después del desastre, es una prioridad que debe guiar nuestro accionar. En situaciones de emergencia y hasta la recuperación, una educación de calidad proporciona la protección física, psicosocial y cognitiva necesaria para mantener y salvar vidas.

La educación, es una de las condiciones que se desarrolla en las políticas públicas de manera consiente que permite el proceso del adelanto institucional. 


\section{Conclusiones}

El ambiente de aprendizaje entendido como un entorno en el cual ocurren ciertas relaciones en la institución educativa, induce a pensar en el aula como un sistema cerrado en el que la información entra con el ingreso del docente, previamente preparado, cuyos conocimientos sirven únicamente para solucionar problemas escolares; en contraste con ello, el ambiente de aprendizaje debe permitir que la vida, la naturaleza y el trabajo ingresen al entorno, como materias de estudio, reflexión e intervención. Esto nos conduce a plantear tres condiciones para el ambiente de aprendizaje, es decir deberán ser: delimitados, estructurados y flexibles.

Los escenarios educativos en el Ecuador han transitado desde una educación poco flexible, standarizada en su totalidad, no inclusiva, selectiva y estratificada hacia una educación cada vez más de equidad, calidad, atención a la más amplia diversidad y participación democrática

Intensiva y centrada en el estudiante y en la relación de actores formativos, potenciando una educación pública de calidad para toda la población, extendiendo el derecho a la educación y las oportunidades formativas en los diversos tramos de la enseñanza y a lo largo de toda la vida.

El cambio educativo depende de la cantidad, calidad y coherencia que se establece entre las distintas relaciones, conexiones y dimensiones, en el que la innovación pedagógica apunta hacia la transformación educativa y social en oposición a los presupuestos neoliberales centrados en la formación unidimensional de trabajadores competentes para el mercado de trabajo, y pone el foco en los valores de la solidaridad, cooperación, desarrollo integral del ser humano y justicia social.

\section{Bibliografía}

Celia Sarango Lapo et al . (Abril de 2018). Prácticas Educativas Abiertas: experiencias de innovación en una institución de educación superior. Guayaquil: Doctorado Universidad de Salamanca.

Edgar Isch López. (2011). Las actuales propuestas y desafíos en educación en el caso ecuatoriano. Educación Social Campinas, 373-391.

Educación, M. d. (2013). Orientaciones para la preparacion y la respuesta de emergencias . Quito: MINEDUC.

Espinosa, B. (2008). Estado social en Ecuador: las politicas y sus transformaciones recientes. Pontificia Universidad Católica del Perú, Centro de investigaciones sociologicas, economicas, politicas y antropologicas . Recuperado el 05 de Diciembre de 2017, de http://repositorio.pucp.edu.pe/index/bitstream/handle/123456789/47382/espinosa.pdf?seq uence $=1$ 
Isch, L. E. (abr.-jun. de 2011). Las Actuales Propuestas Y Desafíos En Educación: El Caso Ecuatoriano. Scielo, 32(115), 373-391. Recuperado el 03 de diciembre de 2017, de http://www.scielo.br/pdf/es/v32n115/v32n115a08.pdf

Paladines, C. (2015). Perspectiva de cambio en la Educación Básica y el Bachillerato en el Ecuador 2007-2013. Praxis, 13-31.

Paladines, C. (Septiembre-Diciembre de 2015). Perspectivas de cambio en la Educación Básica y en el Bachillerato. Ecuador: 2007-2013. Scielo , 19(3), 13-31. doi:ISSN 0328-9702 / ISSN 2313-934X

Sarango, C. (2015). Prácticas Educativas Abiertas: experiencias de innovación en una institución de educación superior del Ecuador. Virtualis Revista de cultura digital, 6(2). Recuperado el Diciembre de 2017, de http://aplicaciones.ccm.itesm.mx/virtualis/index.php/virtualis/article/view/138/172

Sarango, C. (s.f.). Prácticas Educativas Abiertas: experiencias de innovación en una institución de educacion superior del Ecuador.

Vélez, X., Tárraga, R., \& Fernandez, I. (Noviembre de 2016). Formación inicial de maestros en Educación Inclusiva: una comparación entre Ecuador y España. Revista nacional $e$ internacional de Educación Inclusiva , 9(3). doi:ISSN: 1889-4208.; e-ISSN 1989-4643.

Ximena Velez Calvo et al. (2016). Formación inicial de maestros en educacion inclusiva: una comparación entre Ecuador y España. Revista nacional e internacional de educacion inclusiva, 75-90.

Xiomara Velez, R. T. (2016). Formación inicial e Internacional de Educcación Inclusiva. Revista Nacional e Internacional de Educación Inclusiva , 1-20. 